\title{
Modified technique of stent fenestration of the atrial septum
}

\section{O Stümper, M Gewillig, J Vettukattil, W Budts, M Chessa, M Chaudhari, J G C Wright}

See end of article for authors' affiliations ..................

Correspondence to: Dr O Stümper, The Heart Unit, Birmingham Children's Hospital Steelhouse Lane, Birmingham B6 $4 \mathrm{NH}$, UK; oliver.stumper@ bhamchildrens.wmids.nhs.uk

Accepted 20 March 2003

\begin{abstract}
Objectives: To develop a modified technique for stenting the atrial septum in the treatment of patients with a failing Fontan operation or pulmonary hypertension. Setting: Two tertiary referral centres. Study design: Prospective collaborative clinical study.

Patients and methods: A stent was mounted on a standard valvoplasty balloon catheter which was constricted to a predefined diameter by a loop created from a temporary pacing wire. Full balloon inflation created a diabolo shaped stent configuration. The technique was employed in 12 consecutive patients to relieve symptoms of a failing Fontan circulation $(n=6)$ or severe pulmonary hypertension $(\mathrm{n}=6)$.

Results: Ex vivo studies confirmed that a diabolo shaped stent configuration could be achieved using the above technique. Transcatheter stent implantation was successful in all 12 patients. All six Fontan patients showed significant clinical improvement. Right atrial pressure decreased from (mean (SD)) $16.8(2.5)$ to $13.7(1.9) \mathrm{mm} \mathrm{Hg}(p<0.05)$, and arterial oxygen saturation from $92.8(1.8) \%$ to 82.7 $(3.8) \%(p<0.01)$. Six patients underwent successful stent fenestration for treatment of pulmonary hypertension. All stents were patent and stable at the most recent follow up (mean 1.75 (1.05) years). No early or late stent related complications were encountered.

Conclusions: This new technique allows placement of a diabolo shaped stent with a predefined diameter across the atrial septum. The diabolo shape increases stent stability, facilitates recrossing of the stent during future catheter interventions, and ensures medium term stent patency.
\end{abstract}

B alloon dilatation and percutaneous stent fenestration of the atrial septum has been reported in the management of selected patients. ${ }^{1-4}$ However, balloon dilatation on its own often results in early reclosure of the fenestration or excessive desaturation. ${ }^{4-6}$ Difficulties experienced with standard techniques of stent placement include early stent occlusion, stent migration, and problems in adjusting stent size to achieve the desired haemodynamic effect.

From our own and others' past experience we speculated that a diabolo shaped stent configuration would be highly beneficial in both the short and the medium term. A modified technique was designed to place a diabolo shaped stent across the atrial septum or baffle. This novel technique was employed in 12 consecutive patients with either a failing Fontan circulation or significant pulmonary hypertension.

\section{METHODS \\ Technique}

A loop of 3-4 mm diameter was created using a set of temporary epicardial pacing wires. The needle ends were removed, as were the distal $5 \mathrm{~cm}$ length of insulating coating. The two wires were tied together to provide a length of about $90 \mathrm{~cm}$. Using the bare end of the wire a secure double knot was formed over a 10-14 French dilator. The resultant loop was then placed over the mid portion of a standard $15-25 \mathrm{~mm}$ valvoplasty balloon catheter (fig 1A). A standard stent (Johnson \& Johnson P308 or P188, or Jomed $17 \mathrm{~mm}$ ) was gently dilated with the help of the tapered end of the 10-14 French dilator. The stent was then mounted on the valvoplasty balloon, taking care that the loop created from the pacing wire was placed accurately in the centre of the balloon and the stent (fig 1B). The stent was then manually crimped and its stability tested. The mounted stent was delivered through a 11-14 French long Mullins sheath, securing the end of the

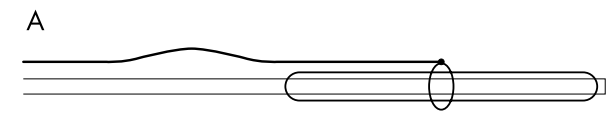

B
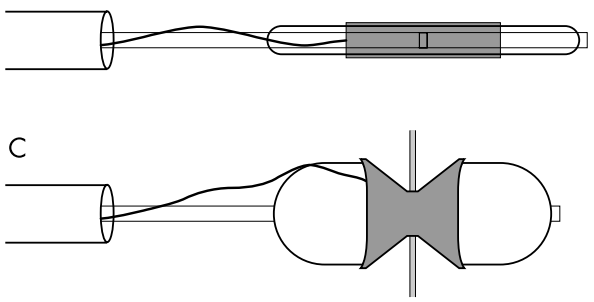

Figure 1 Technique of mounting the stent. (A) A loop of defined diameter is created using a temporary pacing wire. This is placed over a standard balloon valvoplasty catheter. (B) The stent, mounted and crimped with the loop centred in the centre. (C) The stent is placed across the fenestration and the balloon catheter is fully inflated.

temporary pacing wire. The stent was positioned with the radio-opaque loop of pacing wire centred across the atrial septum. The delivery sheath was then withdrawn to the inferior vena cava and the delivery balloon was fully inflated (fig IC).

The technique was initially tested ex vivo using a perforated piece of GoreTex membrane.

\section{Patients}

Twelve patients underwent stent fenestration of the atrial septum between September 1998 and June 2002 (table 1). Procedures were carried out at the Birmingham Children's Hospital, UK, and the University Hospital Leuven, Belgium. Informed consent was obtained in all patients according to the 


\begin{tabular}{|c|c|c|c|c|c|c|c|}
\hline Patient & Diagnosis & Operation & $\begin{array}{l}\text { Interval } \\
\text { (years) }\end{array}$ & Problem & Age (years) & Stent & Follow up (years) \\
\hline 1 & HLHS & fen Fontan & 0.7 & Casts & 4.0 & J\&J P308 & 3.4 \\
\hline 2 & DILV TGA & Fontan & 7.2 & PLE & 13 & J\&J P308 & 2.8 \\
\hline 3 & DILV TGA & Fontan & 2.9 & PLE & 7.4 & J\&J P188 & 2.3 \\
\hline 4 & HLHS & fen Fontan & 0.5 & PLE & 4.6 & Jomed $17 \mathrm{~mm}$ & 0.7 death \\
\hline 5 & TGA & Arterial switch & 7.4 & PHT & 7.5 & J\&JP188 & 1.2 \\
\hline 6 & DILV, TGA, MS & - & & PHT & 16 & J\&J P308 & 0.8 \\
\hline 7 & DORV TGA & Fontan & 2.3 & PLE & 8.3 & J\&J P308 & 3.1 \\
\hline 8 & ccTGA, MA,PA & fen Fontan & 0.1 & LCO & 7.0 & J\&JP188 & 1.9 closure \\
\hline 9 & Primary PHT & - & & PHT & 48 & J\&J P188 & $0.1 \mathrm{HLTX}$ \\
\hline 10 & Primary PHT & - & & PHT & 62 & J\&J P188 & 0.9 death \\
\hline 11 & Primary PHT & - & & PHT & 72 & J\&J P188 & 2.1 \\
\hline 12 & Primary PHT & - & & PHT & 72 & J\&J P188 & 1.8 \\
\hline
\end{tabular}

guidelines of the local ethics committees. Age at intervention ranged from 4.0- 72 years (mean (SD), 26.8 (27.9) years). Six patients suffered from severe complications of the Fontan circulation. Four of these had protein losing enteropathy, one had recurrent bronchial casts (patient 1 ), and one had low cardiac output with persistent chylothorax (patient 8). Intensive standard medical treatment had failed in all. A further five patients (patients 5, 9, 10, 11, and 12) underwent stent fenestration of the atrial septum in the palliative treatment of irreversible pulmonary hypertension. One patient suffered from double inlet left ventricle, progressive mitral stenosis, and increasing pulmonary hypertension (patient 6).

Cardiac catheterisation was done under general anaesthesia using endotracheal intubation. Transoesophageal ultrasound studies were conducted using a dedicated paediatric or adult multiplane probe (General Electric, Sonotron). Heparin 50 units/kg and routine intravenous antibiotics were given. A complete haemodynamic and angiographic study was conducted. Angled radiographic projections were used to achieve best profiling of the atrial septum. Trans-septal puncture was done in eight patients (patients 2, 3, 5, 7, 9, 10, 11, and 12) using a standard Brockenborough trans-septal needle. A 0.035 inch exchange wire was positioned across the atrial septum into the descending aorta or the left upper pulmonary vein. A 11-14 French long Mullins trans-septal sheath was then advanced into the left atrium. The stent was mounted using the technique described above. It was advanced through the sheath until the loop of pacing wire projected onto the atrial septum or baffle. The sheath was then retracted over the balloon catheter back into the inferior vena cava, making sure that the end of the pacing wire was firmly secured outside the sheath. Repeat hand injections of contrast medium through the side arm of the sheath were used to confirm the exact position of the centre of the stent across the fenestration. The balloon was then fully inflated using gentle pressure (fig 2). Following deflation of the balloon the pacing wire and the valvoplasty catheter were removed and repeat haemodynamic and angiographic studies were undertaken. In patients with a Fontan circulation we aimed for an arterial saturation of $80-85 \%$ with $\mathrm{FiO}_{2}<0.3$. In three patients this was not achieved with the initial inflation and further dilatation of the mid section of the stent was done using small valvoplasty balloons. In patients with pulmonary hypertension we aimed to create a fenestration of 4-5 mm. The patients were discharged on oral anticoagulants.

\section{RESULTS}

A diabolo configuration of the stent could be achieved using this technique in all patients (fig 3). Satisfactory positioning of the stent was confirmed on both angiography and transoesophageal ultrasound in all patients. No complications were encountered.

In six patients with a failing Fontan circulation, mean right atrial pressure decreased from 16.8 (2.5) $\mathrm{mm} \mathrm{Hg}$ to 13.7
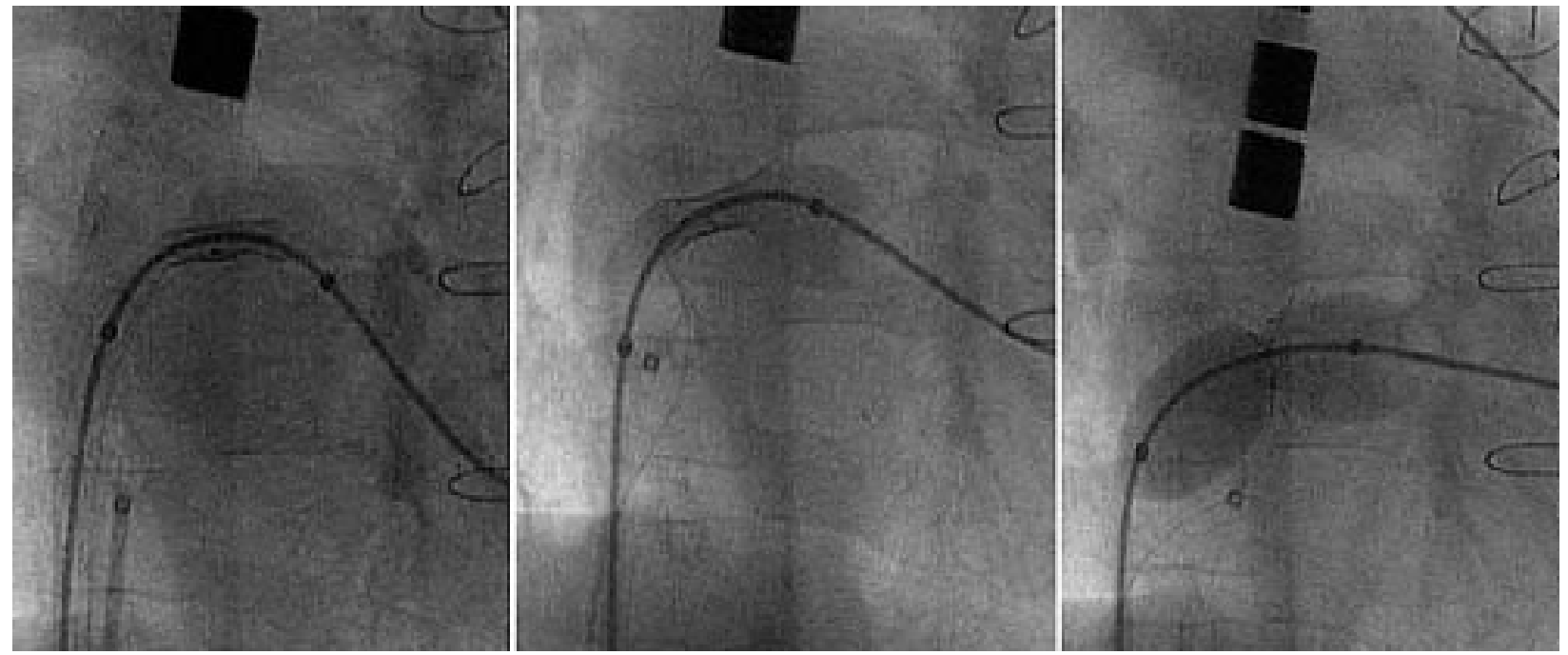

Figure 2 Serial angiographic still frames during stent placement and balloon inflation. 


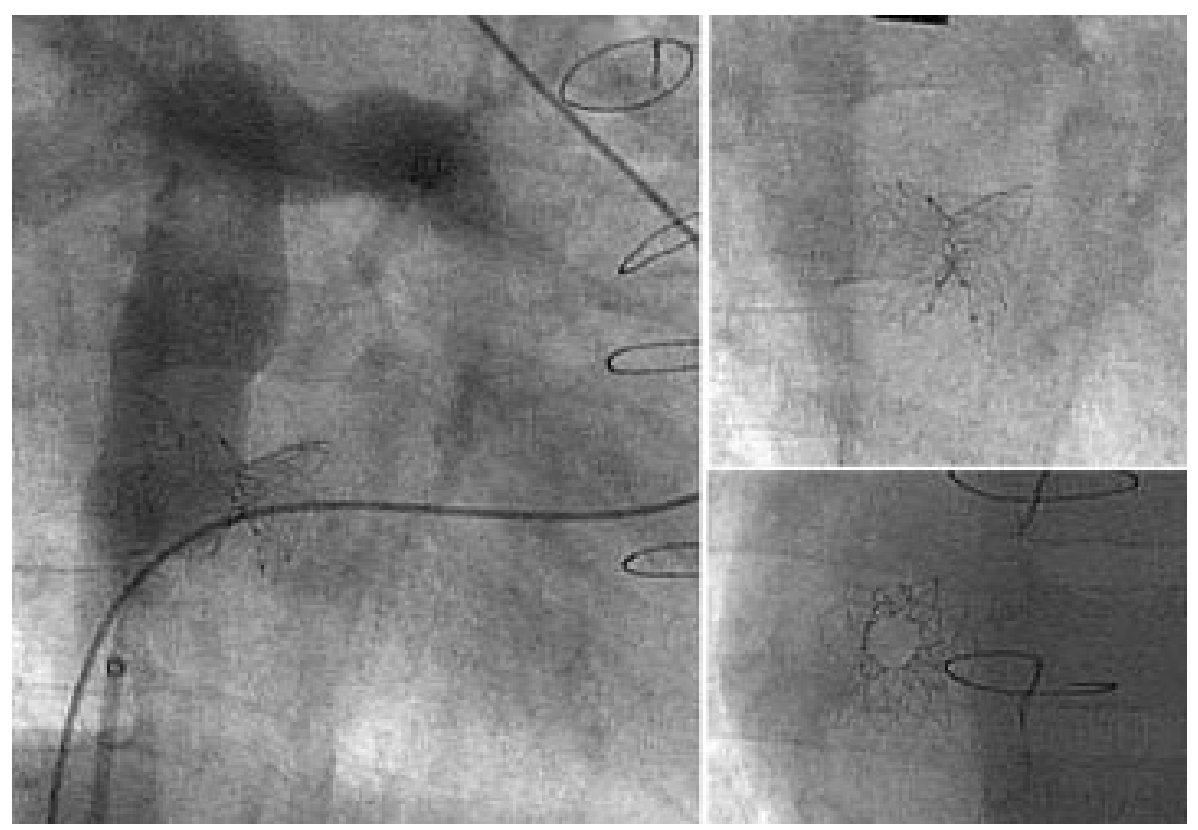

Figure 3 Final stent configuration on fluoroscopy.
(1.9) $\mathrm{mm} \mathrm{Hg} \quad(\mathrm{p}<0.05)$ and arterial oxygen saturation decreased from $92.8(1.8) \%$ to $82.7(3.8) \%(\mathrm{p}<0.01)$ (table 2). All six patients had significant clinical improvement over 7-41 months (mean 28 (11.4) months). Patient 1 underwent transcatheter constriction of the waist of the stent 14 months after the original procedure following lasting resolution of the bronchial casts. This was achieved by the use of an oversized goose neck snare. In patient 2 , the stent was subsequently further dilated because of the recurrence of protein loss. In patient 8 the stent was electively closed 1.9 years after the procedure with a $4 \mathrm{~mm}$ Amplatzer occluder. Patient 4 died suddenly seven months after stent implantation.

The five patients with irreversible pulmonary hypertension have had symptomatic improvement. Patient 9 had immediate significant regression of right heart failure and ascites, facilitating heart and lung transplantation one month after the stent implantation. Patients 10,11 , and 12 had a significant increase in walking distance, from 93 (75) $\mathrm{m}$ to 312 (99) $\mathrm{m}$ following fenestration $(\mathrm{p}<0.001)$.

In patient 6 , with severe mitral stenosis in the context of double inlet left ventricle, the left atrial pressure decreased from $36 \mathrm{~mm} \mathrm{Hg}$ to $17 \mathrm{~mm} \mathrm{Hg}$ after dilatation of the stent to $16 \mathrm{~mm}$ minimum diameter.

All 12 stents were patent and stable at the last follow up at a mean of 21 (12.6) months (range 1-4l months). There was no clinical or echocardiographic evidence of thrombotic complications.

\section{DISCUSSION}

There are few situations in which a restrictive, limited atrial connection or fenestration is advantageous for the patient.
Such situations are right heart failure with low cardiac output, as seen in obstructive pulmonary hypertension, ${ }^{1-3}$ or Fontan circuits with very low cardiac output, excessive venous congestion, or lymphatic problems (protein losing enteropathy, plastic bronchitis, chylothorax, chylopericardium).$^{4-7}$

Fenestration of the atrial septum with subsequent right to left shunt in such situations has the advantage of decreasing the systemic venous pressure and congestion, with an increase in cardiac output. There are, however, clear disadvantages of the new or increased right to left shunt-any increase in cardiac output is obtained with desaturated blood, resulting in cyanosis, and the possibility of paradoxical or septic emboli is created. However, cyanosis is clinically much better tolerated than a low cardiac output.

Fenestration can be achieved by different techniques: by balloon dilatation only, by a stent, or by a fenestrated atrial septal defect occluder.

With balloon dilatation only, a hole is progressively dilated with multiple balloons, increasing in size until the desired clinical result is obtained. This technique is relatively easy. However, disadvantages are multiple - the final size of the connection is difficult to predict; multiple balloons may be needed; and spontaneous closure of such connections is usually observed over days to weeks. ${ }^{8-10}$ Redo dilatation after some weeks with a balloon is dangerous: the fenestration will have fibrous walls, which will usually resist dilatation and only give way (rupture) with very big balloons, resulting in a broad tear and excessive (lethal) right to left shunt. ${ }^{11}$

Because of the unsatisfactory long term results of fenestrations obtained by balloon dilatation only, stent implantation has been advocated. However, the medium term results were

Table 2 Haemodynamic data in patients with failing Fontan procedure

\begin{tabular}{llllll}
\hline Patient & Problem & Sat pre (\%) & Sat post (\%) & RA pre $(\mathrm{mm} \mathrm{Hg})$ & RA post $(\mathrm{mm} \mathrm{Hg})$ \\
\hline 1 & Casts & 93 & 83 & 15 & 12 \\
2 & PLE & 95 & 88 & 18 & 15 \\
3 & PLE & 95 & 83 & 17 & 14 \\
4 & PLE & 91 & 85 & 18 & 14 \\
7 & PLE & 92 & 77 & 13 & 11 \\
8 & LCO & 91 & 80 & 20 & 16 \\
\hline
\end{tabular}

Casts, bronchial casts; LCO, low cardiac output; PLE, protein losing enteropathy; post, after stent fenestration pre, before stent fenestration; RA, right atrial pressure; Sat, haemoglobin saturation. 
limited because of stent occlusion, stent migration, and difficulties in modifying stent diameter after initial placement. ${ }^{12-16}$ We hypothesised that a diabolo shaped configuration of the stent would enhance stent stability and reduce the risk of stent occlusion.

It was crucial to devise a technique that would prevent dilatation of the stent that exceeded a predefined minimum diameter of the waist, and at the same time ensure pronounced flaring of the ends of the stent. To achieve this, external constriction of an oversized delivery balloon catheter was considered the most secure technique. Temporary pacing wires were used to create this loop, because of their small diameter, radio-opacity, and the feasibility of tying secure double knots, thereby ensuring great radial strength without any stretch. Two pacing wires were joined together to provide sufficient length to allow the distal end to be secured outside the delivery sheath at all times.

Other investigators have proposed the use of specifically designed balloons or the recently available balloon-in-balloon for stent delivery. Tests using a standard snare catheter to constrict the delivery balloon showed that this technique was cumbersome and larger delivery sheaths would also have to be used.

An atrial septal occluder with a custom made fenestration can be implanted (Amplatz, AGA, Minneapolis, Minnesota, USA). The septum is first dilated in order to accommodate the atrial occluder. Although this is a tempting technique, it has multiple disadvantages: a large atrial opening is required during the procedure; much foreign material is left in the atrial septum with the risk of thrombus formation; the atrial communication is fixed and cannot be enlarged; and the prosthesis is expensive (list price in 2002, US\$4000 ( £2500/ $€ 4000)$ ).

The technique described here proved successful in the delivery and creation of a diabolo shaped stent across the atrial septum. Initially we used rather long stents of some $30 \mathrm{~mm}$ in length (P308). These shortened significantly with flaring of the ends using an oversized delivery balloon. Using the delivery technique described, the waist of the stent has a great tendency to self centre across the atrial septum or baffle. Thus later on in our experience with the technique we felt confident in using shorter stents ( 17 or $18 \mathrm{~mm}$ length (P188 or Jomed 17)) in order to minimise stent protrusion into the atrial chambers. There were no late stent occlusions in our series. Serial saturation measurements in the group of Fontan patients were stable at the time of writing. Four patients underwent repeat catheterisation. In all cases recrossing of the stent was uncomplicated owing to its diabolo shape. In two cases the waist of the stent was dilated further because of persistent symptoms. In patient 1 the size of the stent was reduced by crimping the waist, using a goose neck snare. In patient 8 the stent was occluded using a small atrial septal defect occluder.

\section{Conclusions}

This modified technique of stent fenestration of the atrial septum is safe and effective and provides good medium term stent patency. The procedure can be a final or temporary palliation before more aggressive treatments like Fontan takedown or heart (or heart-lung) transplantation are considered.

\section{ACKNOWLEDGEMENT}

Sponsored in part by the Belgian Foundation for Research in Paediatric Cardiology.

\section{Authors' affiliations}

O Stümper, J Vettukattil, M Chessa, M Chaudhari, J G C Wright, Birmingham Children's Hospital, Birmingham, UK

M Gewillig,W Budts, Universitair Ziekenhuis, Leuven, Belgium

\section{REFERENCES}

1 Hausknecht M, Sims R, Nihill M. Successful palliation of primary pulmonary hypertension by atrial septostomy. Am J Cardiol $1978 \cdot 42 \cdot 453-7$

2 Rothman A, Sklansky MS, Lucas VW, et al. Atrial septostomy as a bridge to lung transplantation in patients with severe pulmonary hypertension. Am J Cardiol 1999;84:682-6.

3 Takigiku K, Shibata T, Yasui K, et al. Successful blade atrial septostomy in a patient with severe primary pulmonary hypertension - a case report. Jpn Circ J 1997:61:877-81.

4 Mertens L, Dumoulin M, Gewillig M. Effect of percutaneous fenestration of the atrial septum on protein-losing enteropathy after the Fontan operation. Br Heart J 1994;72:591-2.

5 Warnes CA, Feldt RH, Hagler DJ. Protein-losing enteropathy after the Fontan operation: successful treatment by percutaneous fenestration of the atrial septum. Mayo Clin Proc 1996;71:378-9.

6 Kreutzer J, Lock JE, Jonas RA, et al. Transcatheter fenestration dilation and/or creation in postoperative Fontan patients. Am J Cardiol 1997;79:228-32.

7 Mertens L, Hagler DJ, Sauer U, et al. Protein-losing enteropathy after the Fontan operation: an international multicenter study. PLE study group. J Thorac Cardiovasc Surg 1998;1 15:1063-73.

8 Rao PS. Static balloon dilatation of the atrial septum. Pediatr Cardiol 1996;17:349-0.

9 Ayabakan C, Karagoz T, Celiker A. Dilatation of a restrictive interatrial communication using a balloon angioplasty catheter. Turk J Pediatr 2000;42:325-7.

10 Nishimoto K, Keane JF, Jonas RA. Dilation of intra-atrial baffle fenestrations: results in vivo and in vitro. Cathet Cardiovasc Diagn 1994;31:73-8.

11 Henneveld HT, Hutter P, Hitchcock FJ, et al. Catastrophic transcatheter baffle fenestration for failing Fontan physiology. Ann Thorac Surg 1998;65:268-70.

12 Miga DE, Clark JM, Cowart KS, et al. Transcatheter fenestration of hemi-Fontan baffles after completion of Fontan physiology using balloon dilatation and stent placement. Cathet Cardiovasc Diagn 1998:43:429-32.

13 O'Laughlin MP. Stent fenestration of hemi-Fontan baffles: an intriguing addition to the armamentarium. Cathet Cardiovasc Diagn 1998;43:433.

14 Miga DE, Clark JM, Cowart KS, et al. Transcatheter fenestration of hemi-Fontan baffles after completion of Fontan physiology using balloon dilatation and stent placement. Cathet Cardiovasc Diagn 1998:43:429-32.

15 Gewillig M, Mertens L, Stockx L. Percutaneous fenestration of the atrial septum with a stent: an experimental study. Cor Europaeum 1995:4:122-5.

16 Pedra CA, Pihkala J, Benson LN, et al. Stent implantation to create interatrial communications in patients with complex congenital heart disease. Cathet Cardiovasc Intervent 1999;47:310-13. 